\title{
Recurrent Pharyngeal Carcinoma
}

National Cancer Institute

\section{Source}

National Cancer Institute. Recurrent Pharyngeal Carcinoma. NCI Thesaurus. Code C5103.

The reemergence of carcinoma of the pharynx after a period of remission. 